\title{
El clima laboral en la biblioteca universitaria: el caso de seis bibliotecas universitarias en Colombia
}

\author{
Claudia Virginia Becerra-Márquez ${ }^{\star}$ \\ José Javier Bermudez-Aponte*
}

Artículo recibido:

22 de agosto de 2019

Artículo aceptado:

15 de enero de 2020

Artículo de investigación

\section{Resumen}

La investigación se centra en el clima laboral en bibliotecas universitarias de la ciudad de Bogotá, para lo cual se definieron cinco categorías: relaciones laborales, innovación y cambio, recursos y condiciones de trabajo, compensación y remuneración, y orientación e identidad. La metodología que se empleó fue cuantitativa, consistió en la aplicación de un instrumento a 120 empleados pertenecientes a seis bibliotecas. Los resultados evidenciaron un clima laboral estable, siendo la categoría identidad -apropiación de los valores institucionales y la satisfacción del lugar ocupado en la organización- la que obtuvo la mejor percepción; por su parte, las carencias más significativas frente al ambiente laboral en las bibliotecas

* Universidad de La Sabana, Colombia

claudiabm@unisabana.edu.co javier.bermudez@unisabana.edu.co

INVESTIGACIÓN BIBLIOTECOLÓGICA, vol.34, núm. 84, julio/septiembre, 2020, México, ISSN: 2448-8321 pp. 59-77 
están centradas principalmente en temas de innovación y cambio, así como en las relaciones laborales.

Palabras clave: Biblioteca Universitaria; Relaciones Laborales; Universidad; Ambiente Laboral

Working environment in the university library: six Colombian University Libraries

Claudia Virginia Becerra-Márquez and José Javier Bermudez-Aponte

\begin{abstract}
Research centered on work climate in university libraries in Bogotá, for which five categories were defined: labour relations, innovation and change, resources and working conditions, compensation and remuneration, and orientation and identity. The methodology used was quantitative, consisting in the application of an appliance to 120 employees from six libraries. The results showed a stable labour environment, being the identity category -appropriation of institutional values and satisfaction of the place occupied in the organization- the one that obtained the best perception. On the other hand, the most significant disadvantages with regard to the work environment in libraries were mainly centred on innovation and change issues, as well as in labor relations.
\end{abstract}

Keywords: Academic Libraries; Labour Relations; University; Work Environment

\title{
INTRODUCCIÓN
}

$\mathrm{L}$ as bibliotecas universitarias tienen como propósito principal servir de apoyo a los procesos relacionados con las funciones sustantivas de la universidad: docencia, investigación y proyección social, y dentro de su planeación estratégica es necesario comprender las políticas e intereses institucionales, así como crear alianzas y coaliciones que permitan aunar esfuerzos para lograr sus objetivos (Nureña, 2019 y Birdsall, 2006). 
La importancia de la biblioteca para la formación de las comunidades universitarias es una temática ampliamente abordada (Calva González, 2012; Paredes y Pérez, 2018; Gamboa, 2000 y Rodríguez-Bravo et al., 2017), que presenta como una de sus características principales la formación del personal y el clima laboral en el interior de esta unidad.

Incentivar el uso de los recursos de información de las bibliotecas universitarias depende en gran medida del personal que labora en ellas, de allí la importancia de la formación de quienes trabajan en estas unidades (Múnera, 2018); para lograr el éxito de la biblioteca, se debe tener personal comprometido y talentoso (Calva González, 2012).

Los nuevos roles del personal bibliotecario, producto de los cambios en el manejo y acceso a los recursos de información, lo ubican como un actor central para el desarrollo de la biblioteca, sus usuarios y en consecuencia de la universidad (Garloch, 2016); de ahí surge la necesidad de estudiar el ambiente laboral como un factor crítico que media los procesos y servicios ofrecidos por la biblioteca universitaria.

\section{La biblioteca universitaria actual}

Según la American Library Association (ALA), la biblioteca universitaria es "una combinación orgánica de personas, colecciones y edificios cuyo propósito es ayudar a sus usuarios en el proceso de transformar la información en conocimiento" (Gómez, 1998: 363). Esta definición resalta el aporte al conocimiento, ya que la función de la biblioteca universitaria es animar a toda la comunidad educativa en la búsqueda personal e individual del conocimiento y del saber.

Un concepto de biblioteca universitaria ampliamente referenciado es el propuesto por Lihon (1983: 50), quien afirma que la función de la biblioteca universitaria es proveer una adecuada información bibliográfica, teniendo en cuenta la estructura de la universidad, para satisfacer las necesidades de la educación integral de la comunidad universitaria y de las que se originan en la investigación.

Según Balagué (2003), las nuevas tecnologías han creado cambios en los procesos de apropiación y difusión del conocimiento, lo que ha impuesto a las bibliotecas cambios en sus tradicionales metodologías para reinventarse y adaptarse como una herramienta útil de cara a las nuevas tendencias de aprendizaje y a los espacios de enseñanza, así como la convergencia organizativa y la convergencia tecnológica en la biblioteca universitaria.

Este cambio forzoso ha generado nuevas tendencias en las bibliotecas universitarias orientadas a convertirse en centros de recursos, donde confluyen 
la tecnología, las tipologías de información y los espacios, tal como lo afirma Gallo-León (2018: 105): “ya se está produciendo, una liberación del espacio que exigían las colecciones en papel, que pasa a ser destinado a las personas, al intercambio social y a la creación, tanto individual como colectiva”.

El inicio de los cambios de las bibliotecas universitarias se da con la creación en Inglaterra de los Learning Resources Centers (LRC, por sus siglas en inglés); en Italia se denominaron Centri di Risorse per L'apprendimento, en Francia aparecen los Centre de Documentation et D'information, en Estados Unidos los Information Commons, pero es en España donde nace el concepto CRAI (Centros de Recursos para el Aprendizaje y la Investigación).

La propuesta de la Red de Bibliotecas Universitarias Españolas (REBIUN) afirma que la biblioteca es un centro de recursos para el aprendizaje, los CRAI, la docencia, la investigación y las actividades relacionadas con el funcionamiento y la gestión de la universidad o institución en su conjunto (REBIUN, 2003: 14).

Esta nueva mirada implica nuevos roles del bibliotecólogo comprometido a nuevos campos de acción, donde ahora actúa como facilitador de información. Adicionalmente, la aparición de los CRAI replantea la necesidad de redefinir políticas y apuntar hacia una revolución dinámica del papel de la biblioteca universitaria. De hecho, revisar los puntos de vista y las percepciones de los bibliotecarios hace parte de un estudio para mejorar la experiencia de los usuarios de las bibliotecas (Singh y Ovsak, 2014).

En este nuevo panorama, de acuerdo con Codina-Vila e Iñigo (2015), a las bibliotecas les resulta complicado dar respuesta adecuada ante fenómenos como el acceso abierto a la información, o la internacionalización y diversidad de perfiles de investigador, entre otros; de allí que los servicios bibliotecarios dedican más atención en dar apoyo a los investigadores en su rol de autores. También se realizan acompañamientos en procesos de acreditación y en tareas de revisión y validación de la producción científica (Iribarren-Maestro et al., 2015). Este énfasis investigativo que las bibliotecas han realizado implementando estos servicios parece haber dado sus frutos en relación al incremento en el uso de las colecciones (Rodríguez-Bravo et al., 2017).

Todo lo anterior conlleva a que la biblioteca universitaria acoja una actitud proactiva y a implantar técnicas e instrumentos de gestión y aseguramiento de la calidad que le permitan adaptarse a las circunstancias cambiantes y actuar con agilidad (Pinto, Balagué y Anglada, 2007: 380). 


\section{Clima laboral en las bibliotecas}

La cultura organizacional ha tenido diversas definiciones desde la aparición del término. Schein (2010: 12) la precisa como un modelo de supuestos básicos que se descubren y aceptan porque dieron resultados efectivos en un proceso de adaptación en la organización, estos valores básicos orientan los comportamientos del individuo y muestran su influencia en la organización; asimismo, determinan en aspectos como el lenguaje que usa, la ambientación del lugar de trabajo y la apropiación del plan organizacional. En lo que concuerda Gonçalves (2000: 97), quien expuso que el clima organizacional es un fenómeno interviniente que media entre los factores del sistema organizacional y las tendencias motivacionales que se traducen en un comportamiento que tiene consecuencias sobre la organización (productividad, satisfacción, rotación, etcétera).

Méndez (2006: 58) menciona que las variables de motivación, liderazgo, control, toma de decisiones, relaciones interpersonales y cooperación se expresan de acuerdo al proceso de interacción social del colaborador en la estructura organizacional; dichas variables orientan las percepciones, el grado de participación, el comportamiento, la satisfacción y el nivel de eficiencia en el trabajo.

El diagnóstico del clima es una técnica en administración y se ha convertido en el instrumento apto para generar cambios en busca de lograr una mayor efectividad organizacional, ya que consiste en detectar y evaluar las causas que afectan el desempeño de los colaboradores (Chiavenato, 2011).

Un clima laboral saludable y seguro influye de manera positiva en la calidad y la productividad de los colaboradores (Dul y Ceylan, 2010: 12). El clima laboral es un fenómeno interviniente que media entre los factores del sistema organizacional y las tendencias motivacionales que se traducen en un comportamiento que tiene consecuencias sobre la organización: productividad, satisfacción y rotación.

Según Diskienė y Goštautas (2010: 297), la importancia de los estudios sobre valores y actitudes relacionadas con la satisfacción laboral es fundamental, ya que los valores tienen un papel central en la determinación del equilibrio entre individuos y organización. La suposición subyacente es que las personas serán más felices, más motivadas y más satisfechas cuando sus valores son congruentes con los de la organización.

La satisfacción laboral es por naturaleza multifacética, Peng (2014: 75) afirma que diferentes investigaciones han demostrado que la satisfacción laboral se puede clasificar en dos dimensiones: intrínseca y extrínseca. En la primera se refiere a sentido de independencia, responsabilidad y logros 
(Hirschfeld, 2000), la segunda se deriva del ambiente de trabajo y la compensación recibida como salario, condiciones de trabajo, supervisión, compañeros y políticas.

El ambiente laboral en las universidades ha sido ampliamente investigado. Sorcinelli y Near (2016: 59) encontraron que los colaboradores, al considerar el trabajo académico una vocación, trabajan desde casa con horarios flexibles, pero se puede generar estrés cuando el trabajo y sus responsabilidades se entrometen en la vida personal.

En el estudio del ambiente laboral en la biblioteca universitaria sobresale la investigación adelantada por Lau (2007), quien analizó varias experiencias latinoamericanas. Los resultados evidenciaron la necesidad de la mejora en la comunicación, el liderazgo directivo, el reconocimiento, las oportunidades de participación, de crecimiento y equidad, demostrando la importancia de variables institucionales orientadas a la percepción negativa de la labor del bibliotecario en la institución y cómo esta percepción influye en el comportamiento del funcionario e impacta el desarrollo de su trabajo.

La investigación de Lau (2007) finaliza con recomendaciones para mejorar el clima laboral en la biblioteca, como diferenciar las labores por tipología de funcionario con base en su preparación académica, propiciar la capacitación, fortalecer los canales de comunicación interna y lograr mayor compromiso directivo.

En un estudio comparativo de las bibliotecas de la Universidad de São Paulo (Brasil) y la Universidad de Antioquia (Colombia), se concluyó que a los bibliotecarios les gustaría tener un clima que propicie la realización personal en el trabajo y que ofrezca oportunidades de promociones y crecimiento profesional (Delgado, 2006: 113). Las categorías de análisis utilizadas fueron objetivos, estructura, liderazgo, relaciones de trabajo, recompensas, mecanismos de apoyo y propensión al cambio.

El estudio de Delgado (2006: 113) sugiere que las características fundamentales de quienes hacen parte de la biblioteca deberían ser capacidad para gerenciar, combinada con conocimiento técnico; experiencia en su área de actuación, combinada con una visión amplia del trabajo bibliotecario, y competencia integral en la especialidad, unida a una cultura general amplia. Para lograr este perfil es necesario invertir en un aprendizaje continuo, sistemático y personalizado, que conduzca al desarrollo de las competencias y habilidades posibles, de acuerdo con sus características personales y las necesidades de la biblioteca.

En el estudio realizado en los bibliotecarios de la ciudad de Mérida, Yucatán, Vargas Echeverría y Flores Galaz (2019: 166) afirman que estudiar las variables cultura organizacional, satisfacción laboral y desempeño laboral en el 
contexto bibliotecológico permite abordar un campo de estudio poco trabajado pero valioso y que es necesario tener en cuenta otras perspectivas adicionales, tales como los jefes y los usuarios, para enriquecer las investigaciones.

Otra tendencia investigativa relacionada con las bibliotecas universitarias y que guarda relación con el clima laboral son los estudios de sus herramientas digitales, desde investigaciones que se centran en las páginas web para conocer el grado de transparencia (Pacios, 2016) hasta aquéllas que contemplan el desarrollo de herramientas digitales para la evaluación y comparación entre bibliotecas universitarias, como la adelantada por Lázaro-Rodríguez et al. (2018), quienes propusieron el Secaba-Rank Universitarias con indicadores de segundo nivel centrados en la eficiencia con el objetivo de identificar buenas prácticas en las bibliotecas universitarias. En este mismo sentido se encuentra la investigación de Martínez y Caridad (2016), sobre una herramienta metodológica que permite evaluar las características estructurales y dinámicas de las bibliotecas universitarias en el espacio digital.

Se cuenta además con investigaciones centradas en la evaluación de infraestructuras y edificios de biblioteca (Gallo-León, 2017), en la manera de integrar las tecnologías de la comunicación a sus procesos de marketing digital para obtener retorno de la inversión (ROI) (González-Fernández, 2015), en los recursos informáticos bibliotecarios para la competitividad académica entendida como productividad, calidad e innovación (Romo-González et al., 2018), y en la gestión de proyectos (Cobo-Serrano y Arquero-Avilés, 2017).

Por lo tanto, la importancia en la medición del clima laboral tiene que ver con la manera en que éste afecta el comportamiento de los miembros de la organización e influye en su motivación laboral (Schein, 2010).

Para el desarrollo de la presente investigación se definió el clima laboral como el conjunto de atributos de un ambiente de trabajo que son percibidos por las personas que componen la organización y que influyen substancialmente sobre su conducta. De esta definición se desprenden seis características o variables que componen el clima laboral en la biblioteca: Relaciones laborales, Innovación y cambio, Recursos y condiciones de trabajo, Compensación y remuneración, Orientación, e Identidad (Martens, 1987; Vélaz, 1996; Currás Pérez, 2010; Domingo, 2013 y Bernal, 2014).

Las Relaciones laborales se entienden desde la integración y colaboración que se percibe con los compañeros de trabajo y la administración. Esta variable se hace evidente en el fomento del trabajo en equipo, compañerismo con otros empleados y manejo de problemas dentro del área.

La Innovación y cambio se relaciona con la manera en que las opiniones e ideas del personal son consideradas por los directivos e influyen en la gestión de la biblioteca. Se evidencia en la percepción de tener la oportunidad 
de innovar, el desarrollo de nuevas habilidades y la percepción positiva frente a los cambios (Domingo, 2013).

Las condiciones físicas que impactan en el desarrollo de la labor diaria se denominaron Recursos y condiciones de trabajo, y se evidencia en la iluminación, temperatura, flujo de aire, limpieza, seguridad industrial e implementos de trabajo.

La Compensación y remuneración se basa en la satisfacción personal, los sentimientos que genera la labor realizada y los factores que mantienen y dirigen una conducta específica. Se relaciona con la formación constante, recibir retroalimentación del desempeño, percibir como satisfactorio el sueldo recibido, las prestaciones, los ascensos y las promociones (Vélaz, 1996). Las compensaciones laborales se dividieron en directas e indirectas, las primeras se refieren a las condiciones salariales y bonificaciones basadas en el rendimiento, mientras que las segundas a los beneficios sociales representados tanto en reconocimientos no económicos (educación, oportunidades de crecimiento) como en auxilios asociados a la base salarial.

La Orientación se relaciona con la percepción frente al jefe inmediato y su capacidad de liderazgo, compromiso con la institución, comunicación asertiva, solución de problemas y toma de decisiones. También incluye la orientación hacia los usuarios y compañeros del trabajo (Martens, 1987).

Finalmente, la Identidad hace referencia al conocimiento y apropiación de los valores institucionales y los valores de la biblioteca, también se relaciona con el lugar o preponderancia que esta unidad ocupa para la organización (Currás Pérez, 2010).

\section{Metodología}

El escenario geográfico que delimitó el trabajo de investigación fue la ciudad de Bogotá. Para identificar las universidades objeto, se utilizó la plataforma del Sistema Nacional de Información de la Educación Superior (SNIES) del Ministerio de Educación Nacional. La investigación se centró en las Instituciones de Educación Superior (IES) privadas y se eligieron 17 bibliotecas cuyas universidades contaban con acreditación de alta calidad o con certificación de calidad con la International Standarization Organization (ISO). La muestra se compone de seis bibliotecas universitarias con un total de 120 participantes, distribuidos como se detalla en la Tabla 1. 


\begin{tabular}{|c|c|c|c|}
\hline Universidad & Bibliotecas & Colaboradores & $\begin{array}{c}\text { Participantes } \\
\text { por muestra }\end{array}$ \\
\hline Universidad de Los Andes & $\begin{array}{c}\text { 1 General y 3 Centros } \\
\text { de Documentación }\end{array}$ & 81 & $43(53 \%)$ \\
\hline Universidad EAN & 1 General & 25 & $25(100 \%)$ \\
\hline Universidad Santo Tomás & 4 Sedes & 17 & $13(76 \%)$ \\
\hline Universidad Antonio Nariño & 4 Sedes & 12 & $12(100 \%)$ \\
\hline Universidad Manuela Beltrán & 1 Sede & 16 & $11(100 \%)$ \\
\hline $\begin{array}{c}\text { Fundación Universitaria } \\
\text { de Ciencias de la Salud }\end{array}$ & 2 Sedes & 11 & \\
\hline
\end{tabular}

Tabla 1. Descripción de las bibliotecas participantes

A partir de las características del clima laboral se elaboraron 10 preguntas de selección y una pregunta abierta para cada una de las categorías planteadas. Los ítems fueron sometidos a validación de pares en dos etapas: en la primera fue revisado y evaluado por nueve expertos temáticos, quienes sugirieron ajustes relacionados con la redacción, claridad de las preguntas y la estandarización de categorías. En la segunda etapa fue revisado y avalado por cada uno de los directores de biblioteca participantes en el estudio.

El instrumento fue aplicado en línea y de manera anónima, con el fin de minimizar el riesgo de sesgo. Para verificar su confiabilidad psicométrica, específicamente su consistencia interna, se utilizó la técnica alfa de Cronbach. ${ }^{1}$ El valor del coeficiente general fue de 0.874 , cifra que, según el criterio de George y Mallery (1995), es considerada muy buena.

Los resultados al aplicar esta técnica de validación muestran que el instrumento es confiable en cada una de las categorías señaladas, tal como puede observarse en la Tabla 2.

\begin{tabular}{|l|c|}
\hline \multicolumn{1}{|c|}{ Categorías } & Alfa de Cronbach \\
\hline Relaciones laborales & 0.897 \\
\hline Innovación y cambio & 0.822 \\
\hline Recursos y condiciones de trabajo & 0.836 \\
\hline Compensación y remuneración & 0.924 \\
\hline Orientación & 0.923 \\
\hline Identidad & 0.839 \\
\hline
\end{tabular}

Tabla 2. Alfa de Cronbach por categorías

1 El coeficiente alfa fue propuesto en 1951 por Cronbach como un estadístico para estimar la confiabilidad de una prueba, o de cualquier compuesto obtenido a partir de la suma de varias mediciones (Cervantes, 2005: 17). 
La categoría con la mayor confiabilidad fue Compensación y remuneración (0.923), la de menor, Innovación y cambio (0.822); es decir, hay consistencia en las variables evaluadas.

\section{ANÁLISIS DE RESULTADOS}

En cuanto a los datos sociodemográficos más representativos, se evidenció que $51.2 \%$ de colaboradores son hombres, frente a $48.8 \%$ de mujeres, indicando la existencia de un equilibrio de género. El $58.7 \%$ de los colaboradores tiene edades entre 25 y 35 años, lo que indica la existencia de una tendencia hacia colaboradores jóvenes.

El 30.6 \% de los colaboradores son profesionales en bibliotecología, esta cifra evidencia que la biblioteca como unidad académico-administrativa no integra los criterios de profesionalidad para su ingreso. En este mismo sentido, se observó que 57 \% de los colaboradores tienen una antigüedad entre cero y cuatro años en la biblioteca, lo que demuestra una alta rotación de los colaboradores. Este aspecto es relevante si se considera que el dominio de una competencia laboral requiere transitar una curva de aprendizaje cuyos efectos no se relacionan únicamente con el desempeño laboral individual, sino que tienen un impacto significativo en el desarrollo de los procesos que se ejecutan en la biblioteca para apoyar las funciones sustantivas de producción de conocimiento en la universidad.

A partir de la información recolectada se realizaron correlaciones entre los datos sociodemográficos y cada una de las categorías planteadas, utilizando las pruebas T, Anova y Wilcoxon. Se presentan a continuación los resultados más significativos.

Respecto al nivel de estudios (en que el primer grupo son los profesionales en bibliotecología y el otro grupo corresponde a otro nivel de estudios) y la categoría Innovación y cambio, se generó una probabilidad significativa de 0.0137 (con una $p<0.05$ en la prueba de Wilcoxon) que se traduce en que el primer grupo está más orientado a tener características de innovación y cambio. De este hallazgo deriva la importancia de considerar acciones institucionales orientadas a la profesionalización del personal que labora en la biblioteca, contribuyendo al desarrollo de competencias específicas asociadas a las labores propias del cargo.

En cuanto al nivel de estudios (en que el primer grupo son los profesionales en bibliotecología y el otro grupo corresponde a otro nivel de estudios) y la categoría Orientación, se generó una probabilidad significativa de 0.0491 (con una $p<0.05$ en la prueba Wilcoxon), lo cual se interpreta como que 
hay mayor apropiación de aspectos institucionales y liderazgo por parte de los profesionales en bibliotecología, lo que sugiere que éstos conocen y están más apropiados del Proyecto Educativo Institucional; es decir, que de manera más consciente pueden desempeñar sus funciones en coherencia con el horizonte institucional y los fines de la propuesta educativa que se oferta a los estudiantes. A la luz de los resultados y del marco teórico es posible afirmar que los profesionales en bibliotecología que tienen un alto grado de orientación pueden agregar valor a la experiencia de los estudiantes universitarios cuando acceden a los servicios de la biblioteca.

Los resultados se presentan por cada una de las categorías. El criterio de análisis fueron los niveles de satisfacción, los cuales se organizaron de la siguiente manera: Alto 100 \% - 90 \%, Medio $89 \%$ - 60 \%, y Bajo 59 \% - 0 \%. Para las preguntas abiertas se realiza una compilación de respuestas donde se unen grupos según similitud.

\section{Relaciones laborales}

Los resultados generales de esta categoría muestran un nivel medio de satisfacción de $78.4 \%$. Se destacan las buenas relaciones entre los compañeros, con la valoración más alta (93\%), y las respuestas con más baja satisfacción fueron el adecuado manejo de los problemas con $71 \%$ y el balance de las cargas laborales con $65.3 \%$, siendo esta última la más crítica.

Dado que en el marco teórico se resaltó la importancia de la satisfacción del personal de biblioteca en relación con su desempeño, los resultados de esta categoría invitan a reflexionar sobre las acciones de gestión del talento humano que pueden emprenderse para generar un mayor balance en la distribución de las tareas. Asimismo, valdría la pena fortalecer las habilidades blandas del personal de la biblioteca, ya que, según los hallazgos, los aspectos relacionales tienen una clara incidencia en la satisfacción con el clima laboral.

Es decir, ha de prestarse atención tanto a la capacitación técnica, que sin duda es indispensable por el rol que cumple el personal de la biblioteca, como al desarrollo de habilidades blandas (comunicación asertiva, manejo de los problemas, toma de decisiones, capacidad de escucha, entre otras), ya que son ante todo personas que establecen vínculos entre ellas como equipo y que a su vez se relacionan con otras personas (estudiantes, docentes y administrativos). 


\section{Innovación y cambio}

Los resultados generales de esta categoría fueron de un nivel medio de satisfacción con $74 \%$. Se resalta con el puntaje más alto el reconocimiento de los cambios de manera positiva, con $80.1 \%$; sin embargo, dos de las cinco preguntas están por debajo de $70 \%$ de satisfacción: oportunidades de innovación con $62 \%$ y proceso de selección con $68.6 \%$.

Esta categoría incluyó una pregunta abierta de respuesta obligatoria: ¿Qué cambiaría de su puesto de trabajo? El 36.7 \% señaló que no cambiaría nada, mientras que $29.2 \%$ señaló la necesidad de mejoramiento del espacio físico o infraestructura y $13.3 \%$ mejoramiento de los incentivos.

Respecto al mejoramiento del espacio o infraestructura, las respuestas señaladas por los colaboradores se orientan a la poca funcionalidad de los lugares de trabajo, aspecto que es vital ya que el mobiliario y las condiciones físicas del entorno laboral afectan la percepción del clima. En cuanto a la pregunta encaminada a la mejora de los incentivos y ascensos, las respuestas se orientaron a la falta de reconocimiento de las capacidades del personal. Al respecto conviene señalar que los estudios sobre clima han demostrado que la compensación emocional es tan importante como la compensación salarial. De allí que convenga propiciar acciones, mecanismos o programas de reconocimiento al desempeño del personal.

\section{Recursos y condiciones de trabajo}

Los resultados generales de esta categoría arrojaron un nivel medio de satisfacción con $74 \%$. Se resaltan, con el porcentaje más alto, las condiciones de iluminación con $84.3 \%$; no obstante, dos de las cinco preguntas están por debajo de $70 \%$ de satisfacción: temperatura, con $59.5 \%$, y rendimiento del equipo de cómputo, con $64.5 \%$. Estos resultados son coherentes con las observaciones destacadas frente a inconformidades de infraestructura en las bibliotecas, que fueron evidenciadas en las preguntas abiertas de la categoría anterior.

Un aspecto que conviene resaltar de este hallazgo es que la biblioteca como espacio físico es compartida también por los estudiantes y los docentes que acuden a realizar diferentes actividades académicas. Por tanto, esta percepción sobre la temperatura y el rendimiento de los equipos no sólo es un aspecto de mejora con respecto al clima laboral de los trabajadores de la biblioteca, sino que puede ser también un factor para tener en cuenta en relación con la satisfacción de los usuarios. 


\section{Compensación y remuneración}

Los resultados generales determinaron un nivel medio de satisfacción de $72.2 \%$. Se destaca con el mayor puntaje la coherencia de las tareas realizadas con el cargo, con $85.1 \%$. Por otra parte, la satisfacción salarial fue de $56.2 \%$ y con ascensos-promociones de $40.5 \%$, éstas fueron las preguntas con el menor nivel de satisfacción. Se puede inferir entonces que, aunque existe una buena caracterización de la labor acorde con las capacidades de quien la desempeña, no se refleja en la remuneración o en las oportunidades de promoción dentro de la institución. En este punto conviene reflexionar en torno a las posibilidades reales que tiene el personal de biblioteca de las instituciones participantes para desarrollar un plan que incentive la cualificación permanente y el acceso a mejores condiciones laborales.

\section{Orientación}

Los resultados generales arrojaron un nivel medio de satisfacción de $85.5 \%$. La percepción más alta se encuentra en el reconocimiento de la calidad como prioridad en su labor, con $92.6 \%$; la respuesta por debajo de $80 \%$ de satisfacción fue la animación al trabajo autónomo con 79.4 \%. Esta categoría también incluyó dos preguntas abiertas de respuesta obligatoria.

Para la pregunta ¿Cómo podría mi jefe inmediato mejorar de manera integral?, $38.3 \%$ señaló que no cambiaría nada, $20 \%$ la necesidad de mejorar en la comunicación, $10 \%$ la necesidad de realizar seguimientos, y $31.7 \%$ hizo otro tipo de referencia a la mejora integral.

Para la pregunta ¿Cómo podría yo mejorar de manera integral?, $25.8 \%$ afirmó que fortaleciendo las propias competencias, lo que indica el deseo de ampliar la formación y capacitación. El 12.5 \% señaló que mejorando la capacidad de servicio. Las respuestas se orientan a satisfacer las necesidades de información de los estudiantes ofreciendo un mejor servicio y brindando siempre diferentes opciones de apoyo y de guía a las necesidades de los estudiantes, ofreciendo asimismo una solución a sus inconvenientes.

Se evidencia también el interés de un porcentaje considerable de participantes para continuar desarrollando sus competencias profesionales.

\section{Identidad}

Los resultados generales de esta categoría arrojaron un nivel medio de satisfacción de $86.4 \%$. Se resalta con un mayor puntaje el conocimiento de 
objetivos y metas por parte de los colaboradores con $93.4 \%$, y con el menor puntaje la participación en la construcción de esos objetivos que arrojó un $70.2 \%$.

El instrumento finalizó con un espacio para que los encuestados manifiesten alguna observación que pueda impactar positivamente el ambiente laboral. De los 120 encuestados, respondieron en este espacio 29 colaboradores, lo que corresponde a $24.1 \%$ de la muestra total. En las respuestas se resaltan como aspectos fundamentales las buenas relaciones interpersonales que deben mantenerse entre compañeros, pero también con los líderes y/o jefes inmediatos. Otro aspecto relevante se orienta a la manifestación de la falta de reconocimiento y estímulos que no permiten generar crecimiento en la institución.

Los hallazgos ratifican lo encontrado en otras dimensiones con respecto a la necesidad de que el personal que labora en las bibliotecas reciba capacitación para el desarrollo de habilidades blandas, las cuales pueden tener impacto positivo tanto en su gestión como en su relacionamiento con compañeros de trabajo y con los usuarios.

Asimismo, dentro del plan de incentivos y estímulos podrían incluirse reconocimientos que incrementen la percepción sobre el valor que la institución otorga al trabajo de quienes impulsan la calidad académica con el acceso a materiales y recursos para la docencia, la consulta y la investigación.

\section{CONCLUSIONES Y RECOMENDACIONES}

En coherencia con las apreciaciones que se encontraron en las diferentes fuentes bibliográficas consultadas, se confirmó la validez de reconocer al ser humano como componente fundamental para la viabilidad y el éxito de los resultados institucionales, pues un colaborador satisfecho mantiene un vínculo fuerte y agrega valor a los procesos institucionales.

En términos generales, se observó un clima laboral estable; la categoría Identidad obtuvo la mejor percepción, particularmente en la apropiación de los valores institucionales y la satisfacción del lugar ocupado en la organización. Esta particularidad debe aprovecharse para impulsar temas relacionados con el mejoramiento del sentido de pertenencia y el crecimiento de la biblioteca; sin embargo, estas tendencias se consolidan en la medida en que se forme una comunidad de colaboradores constantes y motivados, que ligado a procesos de formación genera retención del personal, minimizando la rotación. 
En contraste, la categoría con percepción más baja fue Compensación y remuneración, que no sólo hace referencia a componentes salariales, sino también al manejo de reconocimientos en público, felicitaciones o el apoyo para el desarrollo a través de la formación. En este sentido, se propone la necesidad de crear una ruta de formación específica para los colaboradores, ya que esta clase de estrategias permite crear lazos de interdependencia y responsabilidad, asumir el desarrollo de nuevas ideas orientadas a la innovación y cambio y delegar con confianza habilidades que, indudablemente, impulsan el crecimiento y la competitividad no sólo de la biblioteca sino de toda la institución.

Por su parte, la categoría Innovación y cambio obtuvo baja satisfacción por la falta de oportunidades para generar cambios y no sentir apoyo para las ideas propuestas. Este aspecto contrasta con uno de los resultados de la investigación, teniendo en cuenta que 28 \% de los colaboradores son estudiantes de bibliotecología y son quienes, en su ejercicio académico, se enfrentan a las nuevas tendencias de la profesión y que generalmente aportan nuevas ideas en su ejercicio laboral. Por ello, abrir espacios de práctica académica con estudiantes sobre proyectos institucionales que generen impacto es una estrategia que, además de brindar confianza y sentido de pertenencia en los colaboradores, le permite a la biblioteca estar a la vanguardia en las nuevas tendencias bibliotecológicas.

Otro aspecto que obtuvo baja satisfacción son los Recursos y condiciones de trabajo (elementos físicos, técnicos y tecnológicos con los que se cuenta), por ello se sugiere la realización de estudios ergonómicos que ayuden a evidenciar las deficiencias en condiciones de trabajo.

La carencia de estudios sobre el clima en las bibliotecas colombianas demuestra la necesidad de abordar esta temática ampliamente, ya que el clima influye en el rendimiento de los colaboradores y en el cumplimiento de los objetivos de esta unidad que apoya transversalmente a la universidad.

Aunque la investigación se centró en seis categorías, pueden incluirse en estudios futuros temáticas relacionadas con el ser, como versatilidad, dinamismo y libertad, atributos inherentes al ser humano que pueden generar nuevas perspectivas y líneas de desarrollo que apalanquen el fortalecimiento del clima laboral. 


\section{REFERENCIAS}

Balagué, Nuria. 2003. "La biblioteca universitaria, centro de recursos para el aprendizaje y la investigación: una aproximación al estado de la cuestión en España”. Trabajo presentado en las Jornadas REBIUN: Los Centros de Recursos para el Aprendizaje y la Investigación, Palma de Mallorca, España, mayo.

https://biblioteca.uam.es/sc/documentos/Jornadas_REBIUN/3\%20-\%20biblioteca_universitaria_CRAI.pdf

Bernal, Idolina. 2014. "El clima organizacional y su relación con la calidad de los servicios públicos de salud: diseño de un modelo teórico". Estudios Gerenciales 12: 8-19. https://doi.org/10.1016/j.estger.2014.08.003

Birdsall, Douglas. 2006. Strategic Planning in Academic Libraries: A Political Perspective, American Library Association. Association of College y Research Libraries (ACRL). http://www.ala.org/acrl/publications/booksanddigitalresources/booksmonographs/pil/pil49/birdsall

Calva González, Juan. 2012. "Gestión y desarrollo de recursos humanos en bibliotecas”. Investigación Bibliotecológica 26 (58): 241-245.

Cervantes, Víctor. 2005. "Interpretaciones del coeficiente Alpha de Cronbach". Avances en Medición 3 (1): 9-28.

Chiavenato, Idalberto. 2011. Administración de Recursos Humanos: El capital bumano de las organizaciones, 9a ed. México: McGraw Hill.

Chuaqui, Benedicto. 2002. "Acerca de la historia de las universidades". Revista Chilena de Pediatría 73 (6): 583-585. https://doi.org/10.4067/S0370-41062002000600001

Cobo-Serrano, Silvia y Rosario Arquero-Avilés. 2017. "La gestión de proyectos en las bibliotecas universitarias: percepciones de los profesionales latinoamericanos". Revista General de Información y Documentación 27 (1): 47-260. https://doi.org/10.5209/RGID.56582

Codina-Vila, Mikel y Ruth Iñigo. 2015. "De la investigación al investigador. Adaptando servicios en la Biblioteca Rector Gabriel Ferraté”. El Profesional de la Información 24 (5).

https://doi.org/10.3145/epi.2015.sep.13

Currás Pérez, Rafael. 2010. "Identidad e imagen corporativas: revisión conceptual e interrelación”. Teoría y praxis 7: 9-34.

Delgado, Nora. 2006. "Diagnóstico sobre las preferencias de clima organizacional de los bibliotecarios universitarios: el caso de las universidades de Sao Paulo y Antioquia". Revista Interamericana de Bibliotecología 29 (2): 99-117.

Diskienė, Danuta y Vytautas Goštautas. 2010. "Relationship between individual and organizational values and employees job satisfaction". Current Issues of Business and Law 5 (2): 295-319.

Domingo, Carlos. 2013. El viaje de la innovación. Barcelona: Planeta.

Dul, Jan y Canan Ceylan. 2010. "Work environments for employee creativity”. Ergonomic 54 (1): 12-20.

https://doi.org/10.1080/00140139.2010.542833

Fisher, Colin. 2004. "The Dynamics of Modernization and Job Satisfaction in the British National Health Service". Review of Public Personnel Administration 24 (4): 304-318. 
Gallo-León, José Pablo. 2017. "La evaluación de infraestructuras y edificios de biblioteca: Faulkner-Brown frente al cuestionario de la IFLA". Investigación Bibliotecológica: archivonomía, bibliotecología e información 31 (72): 81-111. https://doi.org/10.22201/iibi.0187358xp.2017.72.57825

Gallo-León, José-Pablo. 2018. "Los cuatro espacios: un modelo para la organización física de la biblioteca”. Anuario ThinkEPI 12: 104-112. https://doi.org/10.3145/thinkepi.2018.11

Gamboa, Sonia. 2000. "Nuevo rol para el profesional de la biblioteca del futuro". Trabajo presentado en el Panel Información en la nueva era, Universidad Nacional Mayor de San Marcos, Lima, Perú. http://hdl.handle.net/10760/5601

Garloch, Lorene. 2016. "Overcoming Library Illiteracy”. The Journal of Higher Education 13 (3): 124-128. https://doi.org/10.1080/00221546.1942.11773264

George, Darren y Paul Mallery. 1995. SPSS/PC+ step by step: A simple guide and reference. New York: Wadsworth Publ. Co.

Gómez, José. 1998. "La biblioteca universitaria”, Manual de Biblioteconomía, editado por Luisa Orera, 363-376. Madrid: Síntesis.

González-Fernández, Nieves. 2015. "ROI en medios sociales: Campañas de marketing en bibliotecas”. El Profesional de la Información 24 (1): 22-30. https://doi.org/10.3145/epi.2015.ene.03

Gonçalves, Alexis. 2000. Fundamentos del clima organizacional. Hampton, USA: Sociedad Latinoamericana para la Calidad (SLC).

Hirschfeld, Robert. 2000. "Does revising the intrinsic and extrinsic subscales of the Minnesota Satisfaction Questionnaire Short Form make a difference?”. Educational and Psychological Measurement 60 (2): 255-270. https://doi.org/10.1177/00131640021970493

Iribarren-Maestro, Isabel, Teresa Grandal, María Alecha, Ana Nieva y Teresa San-Julián. 2015. "Apoyando la investigación: nuevos roles en el servicio de bibliotecas de la Universidad de Navarra”. El Profesional de la Información 24 (2): 131-137. https://doi.org/10.3145/epi.2015.mar.06

Lau, Jesús, coord. 2007. Ambiente laboral: estrategias para el trabajo efectivo en bibliotecas. México: Alfagrama.

Lázaro-Rodríguez, Pedro, Javier López-Gijón, Sergio Alonso, María Martínez-Sánchez y Enrique Herrera-Viedma. 2018. "Secaba-Rank, herramienta online para analizar y evaluar bibliotecas”. El Profesional de la información 27 (2): 278-288. https://doi.org/10.3145/epi.2018.mar.06

Lihon, Danilo. 1983. Planeamiento, organización y administración de centros de documentación. Lima: INIDE.

Lozano, Roser. 2009. "Gestión del cambio y cultura organizacional en la biblioteca pública”. Educación y biblioteca: Revista mensual de documentación y recursos didácticos 21 (170): 101-106.

Martens, Rainer. 1987. Coaches Guide to Sport Psychology. Champaign: Human Kinetics.

Martínez, Sara y Mercedes Caridad. 2016. "Bibliotecas universitarias y cultura digital: indicadores y validación a partir del ranking de Shanghai”. Opción 32 (8): 138-155. 
Méndez, Carlos. 2006. Clima organizacional en Colombia. Bogotá: Universidad del Rosario.

Múnera, María. 2018. “Algunas tendencias de la formación bibliotecológica en América Latina”. Investigación Bibliotecológica: archivonomía, bibliotecología e información 32 (74): 101-122. https://doi.org/10.22201/iibi.24488321xe.2018.74.57912

Nureña, César. 2019. "Bibliotecas universitarias y proyección social: diferencias y extremos en América Latina”. Investigación Bibliotecológica: archivonomía, bibliotecología e información 33 (80): 117-132. https://doi.org/10.22201/iibi.24488321xe.2019.80.58009

Olvera-Lobo, María-Dolores y Belarmina Benítez-de-Vendrell. "Aproximación a las actitudes y percepciones de los usuarios ante las tecnologías de la información”. El Profesional de la Información 17 (2): 199-204. https://doi.org/10.3145/epi.2008.mar.10

Pacios, Ana. 2016. "Universidades transparentes con bibliotecas transparentes". Investigación Bibliotecológica: archivonomía, bibliotecología e información 30 (70): 105-128. https://doi.org/10.1016/j.ibbai.2016.10.006

Paredes, Esther y Rodolfo Pérez. 2018. "La calidad de la biblioteca universitaria y sus ventajas para la formación en educación superior”. Revista Cubana de Educación Médica Superior 32 (1): 1561-2902. http://www.ems.sld.cu/index.php/ems/article/view/1262/651

Peng, Yu-Ping. 2014. "Job satisfaction and job performance of university librarians: A disaggregated examination”. Library and Information Science Research 36:74- 82. https://doi.org/10.1016/j.lisr.2013.02.006

Pérez, Yudith y Milanés Yusnelkis. 2008. "La biblioteca universitaria: reflexiones desde una perspectiva actual". ACIMED 18 (3): 1-39.

Pinto, María, Nuria Balagué y Luis Anglada. 2007. "Evaluación y calidad en las bibliotecas universitarias: experiencias españolas entre 1994-2006”. Revista Española de Documentación Científica 30 (3): 364-383. https://doi.org/10.3989/redc.2007.v30.i3.390

REBIUN (Red de Bibliotecas Universitarias Españolas). 2003. Plan estratégico: 2003-2006. http://hdl.handle.net/10261/74804

Rodríguez-Bravo, Blanca, María Simóes, María Vieira-De-Freitas y José Frías. 2017. "Descubrimiento de información científica: ¿todavía misión y visión de la biblioteca académica?”. El Profesional de la Información 26 (3): 464-479. https://doi.org/10.3145/epi.2017.may.13

Romo-González, José, Laura Murguía, Javier Tarango y Juan Machin-Mastromatteo. 2018. "Evaluación de impacto de los recursos informativos bibliotecarios en la competitividad académica mediante ecuaciones estructurales". Ibersid 12 (1): 43-49.

Schein, Edgar, 2010. Organizational culture and leadership. San Francisco: Jossey-Bass.

Singh, Rajesh y Amber Ovsak. 2014. "Library Experience Matters! Touchpoints to Community Engagement". Journal of Library Administration 53 (5-6): 344-358. https://doi.org/10.1080/01930826.2013.876826

Sorcinelli, Mary y Janet Near. 2016. "Relations between Work and Life Away from Work among University Faculty”. The Journal of Higher Education 60 (1): 59-81. https://doi.org/10.1080/00221546.1989.11778824 
Vargas Echeverría, Shilia Lisset y Mirta Margarita Flores Galaz. 2019. “Cultura organizacional y satisfacción laboral como predictores del desempeño laboral en bibliotecarios”. Investigación Bibliotecológica: archivonomia, bibliotecología e información 33 (79): 149-176.

https://doi.org/10.22201/iibi.24488321xe.2019.79.57913

Vélaz, Juan. 1996. Motivos y motivación en la empresa. Madrid: Díaz de Santos.

Para citar este texto:

Becerra-Márquez, Claudia Virginia y José Javier Bermudez-Aponte. 2020.

"El clima laboral en la biblioteca universitaria: el caso de seis bibliotecas universitarias en Colombia”. Investigación Bibliotecológica: archivonomía, bibliotecología e información 34 (84): 59-77.

http://dx.doi.org/10.22201/iibi.24488321xe.2020.84.58165 